\title{
A facile and novel route for dual-template method synthesis of mesoporous silica material Al-Ce-SBA-15
}

Meng $\mathrm{Li}^{\mathrm{a}}$, Ning Liu ${ }^{\mathrm{a}}$, Zhimin $\mathrm{Wu}^{\mathrm{a}}$, , $*$, Yongfei $\mathrm{Li}^{\mathrm{a}, \mathrm{c}}$, Shanshan $\mathrm{Li}^{\mathrm{a}}$, Wenzhi $\mathrm{Xu}^{\mathrm{a}}$, Zhantao $\mathrm{Luo}^{\mathrm{a}}$, Yuejin $\mathrm{Liu}^{\mathrm{a}, \mathrm{b}, *}$

${ }^{a}$ School of Chemical Engineering, Xiangtan University, Xiangtan 411105, China

${ }^{\mathrm{b}}$ National \& Local United Engineering Research Center for Chemical process

Simulation and Intensification, Xiangtan University, Xiangtan 411105, China

${ }^{\mathrm{c}}$ Department of Chemical Engineering, University of Massachusetts Amherst, MA

01003, USA

*Corresponding authors. Tel: +86-731-58293549

E-mail addresses: xdwuzm@163.com (Z.Wu), xdlyj@163.com (Y. Liu).

\section{Abstract}

A kind of ordered mesoporous material Al-Ce-SBA-15 was successfully prepared by the method of dual-templates $\mathrm{CTAB}$ and $\mathrm{P} 123$ with adding appropriate amount of $\mathrm{Al}$ through a one-step hydrothermal synthesis. The prepared Al-Ce-SBA-15 material was characterized by XRD, EDX, HRTEM, SEM, FT-IR and $\mathrm{N}_{2}$ adsorption-desorption isotherm technique. The results showed that the heteroatoms $\mathrm{Al}$ and $\mathrm{Ce}$ highly dispersed on the surface of SBA-15 and the prepared Al-Ce-SBA-15 material still retained a desirable ordered mesoporous structure, high specific surface area, large pore volume and narrow pore size distribution. Especially, a uniform coating layer was formed on SBA-15, and the aggregation of metal Ce was avoided by adding appropriate amounts of Al.

Keywords: Porous materials, Aggregation, Al-Ce-SBA-15, Dual template,

Microstructure

\section{Introduction}

The ordered mesoporous silica SBA-15 material received great application due to its important characteristics of high surface areas, uniformed mesoporous size and better stability [1-3]. The multi-functionalization modified SBA-15 with the shape similar to 
SBA-15 have widely used in adsorption, separation and catalysis [4-6]. Therefore, there are many studies aimed at modifying the mesoporous materials in order to increase the potential applicability $[4,5]$. However, the mesostructure of the modified SBA-15 are easily damaged when some strong basic species is introduced by means of high-temperature calcination of the corresponding precursor [7]. It is an effective strategy to disperse some metal oxides, such as $\mathrm{Al}_{2} \mathrm{O}_{3}, \mathrm{MgO}$ and rare earth metal oxides, onto the surface of mesoporous SBA-15 followed by calcination to form a compact coating layer [8-10]. Ce is a common rare earth element which can form metal oxide layers on the surface of SBA-15, and it also can modulate the hydrothermal and thermal stability of mesoporous silica materials $[11,12]$. However, there are some agglomerate phenomena of metal Ce species on surface of SBA-15, when they were introduced by post-synthesized method [6]. Moreover, little attention has been paid to how to solve the agglomerate phenomena of metal Ce species on mesoporous material surface.

In our pre-experiments, the agglomerate phenomena of metal $\mathrm{Ce}$ species have been alleviated to a certain extent by using dual-template method for preparing the mesoporous silica material of Ce-SBA-15. In order to effectively eliminate the metal Ce agglomeration on SBA-15, the method of dual-templates CTAB and P123 with adding appropriate amount of $\mathrm{Al}$ was adopted through one-step hydrothermal synthesis for Al-Ce-SBA-15. Hence, the interaction of $\mathrm{Ce}$ and $\mathrm{Al}$ species firstly directly introduced on the surface of the preformed SBA-15 mesoporous material of Al-Ce-SBA-15 was successfully prepared using dual-templates. In this method, a uniform coating layer was formed on SBA-15, and the aggregation of metal Ce was avoided.

\section{Experimental}

Synthesis of ordered mesoporous silica Al-Ce-SBA-15 material: 2.988 g CTAB, 3.96 $\mathrm{g} \mathrm{Ce}\left(\mathrm{NO}_{3}\right)_{3} \cdot 6 \mathrm{H}_{2} \mathrm{O}$ and $0.428 \mathrm{~g} \mathrm{Al}\left(\mathrm{NO}_{3}\right)_{3} \cdot 9 \mathrm{H}_{2} \mathrm{O}$ were dissolved in $43.5 \mathrm{ml}$ of alcohol solution with stirring at $40{ }^{\circ} \mathrm{C}$ for $24 \mathrm{~h}$. After crystallization at $110{ }^{\circ} \mathrm{C}$ for $5 \mathrm{~h}$, the sample I was obtained. $4.39 \mathrm{~g}$ TEOS was dissolved in $65.17 \mathrm{ml}$ of $1.6 \mathrm{M} \mathrm{HCl}$ solution containing $2.07 \mathrm{~g} \mathrm{P} 123$ with stirring at $40{ }^{\circ} \mathrm{C}$ for $24 \mathrm{~h}$, the sample II was obtained. The 
sample I mixed with the sample II with vigorously stirring at $40{ }^{\circ} \mathrm{C}$ for $24 \mathrm{~h}$ and then was crystallized for $48 \mathrm{~h}$ at $100{ }^{\circ} \mathrm{C}$. The obtained product was filtered, washed with alcohol solution and dried at $60{ }^{\circ} \mathrm{C}$. The Al-Ce-SBA-15 material was prepared by calcination at $550{ }^{\circ} \mathrm{C}$ for $6 \mathrm{~h}$. For comparison, Ce-SBA-15 material was prepared following the above similar steps except that $\mathrm{Al}\left(\mathrm{NO}_{3}\right)_{3} \cdot 9 \mathrm{H}_{2} \mathrm{O}$ was not added.

Characterizations: X-ray diffraction patterns of calcined samples recorded on a $\mathrm{D} / \max 255018 \mathrm{~kW}$ rotating anode X-ray diffractometer with the monochromatic $\mathrm{Cu} \mathrm{K} \alpha$ radiation. Specific surface area was measured by a Quantachrome's NOVA-2100e Surface Area instrument by physisorption of nitrogen at $-196{ }^{\circ} \mathrm{C}$. SEM images were performed on a Hitachi SU8000 scanning electron microscope. The pore structures were established by the HRTEM experiments on a JEM-2010 UHR electron microscope operated at $200 \mathrm{kV}$. FT-IR spectra were recorded on a Nicolet Nexus 470 spectrometer using $\mathrm{KBr}$ pellets. EDX mapping images were captured on a Tecnai G2 F20 S-TWIN atomic resolution analytical microscope.

\section{Results and discussion}

Fig. 1A shows the small-angle X-ray diffraction patterns of Ce-SBA-15, Al-Ce-SBA-15 and SBA-15. All the samples exhibited three peaks which correspond to the characteristic reflections (100), (110) and (200) of 2-D hexagonal lattice symmetry. It indicates the existence of the mesostructures of the samples. In general, the fwhms of the (100) reflection should increase with the introduction of metal oxides because of the decreasing scatter contrast between pore walls and pore space. However, the fwhms of the (100) reflection of both Ce-SBA-15 and Al-Ce-SBA-15 samples are apparently smaller than that of SBA-15. This abnormal phenomenon might be ascribed to the formation of a smooth Ce or Al-Ce layer on the surface of SBA-15, which is similar to what happened in the magnesia-modified SBA-15 [13]. Through a comparison with the reference result [6], it could demonstrate that the well-ordered pore structure was retained. The intensities of the diffraction peaks (100), (110) and (200) decreased slightly, which probably is attribute to the introduction of $\mathrm{Al}$ and $\mathrm{Ce}$ species to cause a 
slight destruction of the mesostructured SBA-15.

Fig. 1B shows the FT-IR absorption spectra of SBA-15, Ce-SBA-15 and Al-Ce-SBA-15. The absorption band at $967 \mathrm{~cm}^{-1}$ is assigned to the stretching vibrations of $\mathrm{Si}-\mathrm{O}-\mathrm{Si}$ or $\mathrm{Si}-\mathrm{O}-\mathrm{H}$ presenting in the framework of SBA-15 [14]. Typically, the shifting of the absorption band toward the lower wavenumber is considered as the indication for incorporating of hetero species into the framework of silica tetrahedral [15]. However, there is no significant change in intensity at $967 \mathrm{~cm}^{-1}$ in the FT-IR spectra of Ce-SBA-15 and Al-Ce-SBA-15, which suggested that Al and Ce species have not been into the framework of SBA-15. EDX analysis was performed to estimate the composition of Al-Ce-SBA-15 samples, in which both Ce and Al signals emerged in Fig. 1C. The resultant $\mathrm{Si} / \mathrm{Al}$ and $\mathrm{Si} / \mathrm{Ce}$ atomic ratios are similar, which indicates a comparatively good distribution of $\mathrm{Ce}$ and $\mathrm{Al}$ inside the samples. Combining XRD, FT-IR, EDX results, Al and Ce species are highly dispersed and formed a uniform coating layer on the surface of SBA-15.
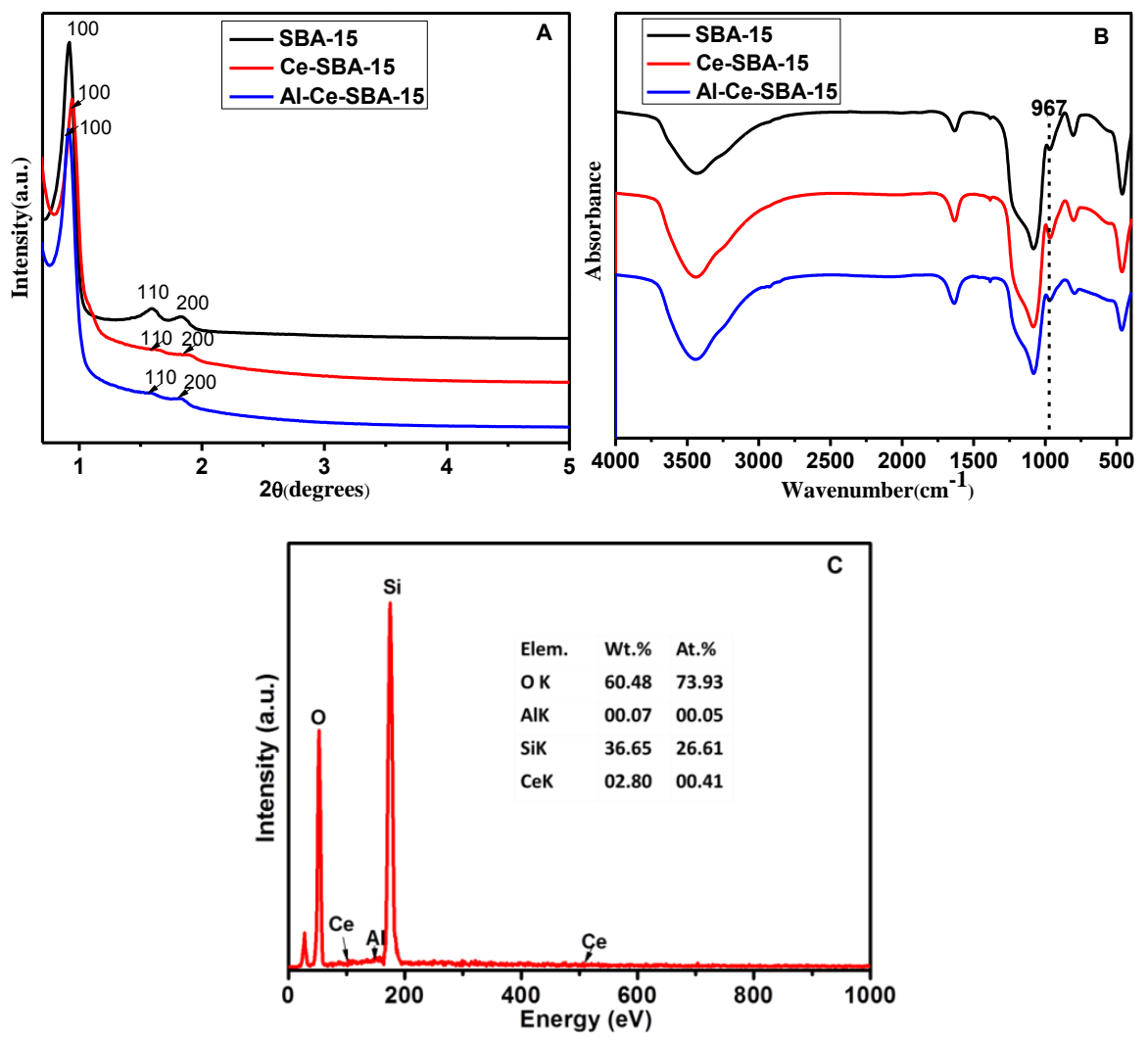
Fig. 1. (A) Low-angle XRD patterns and (B) FT-IR spectra of SBA-15, Ce-SBA-15, Al-Ce-SBA-15, and (C) EDX of Al-Ce-SBA-15.

Fig. 2A and Fig. 2B show the TEM images of Ce-SBA-15 and Al-Ce-SBA-15. These mesoporous silica materials exhibit an ordered mesostructure with the hexagonal ordered array of one dimensional mesoporous channel. However, some spots appeared on the surface of mesoporous materials (Fig. 2A), which indicates that there are some agglomerations of Ce on the surface of Ce-SBA-15. These spots disappeared after Al is introduced into the Ce-SBA-15. It means no crystal particles of $\mathrm{Al}$ or/and Ce existed on the surfaces of Al-Ce-SBA-15(Fig. 2B). Moreover, the pores of the catalysts are open, not blocked, suggesting that the Al and Ce species well dispersed on the supports. Therefore, it can conclude that the aggregation of metal Ce can be avoided by adding appropriate amounts of Al. The SEM images (Fig. 2C and 2D) of all the samples exhibited a fiber-like uniform cylindrical morphology, which is in good agreement with the typical SBA-15 morphology [16]. Combining XRD, FT-IR, EDX and TEM results, the Al-Ce-SBA-15 has higher order and regularity mesostructured than Ce-SBA-15, which may be attributed to improvement of the compactness of mesoporous silica materials by adding the $\mathrm{Al}$ species.

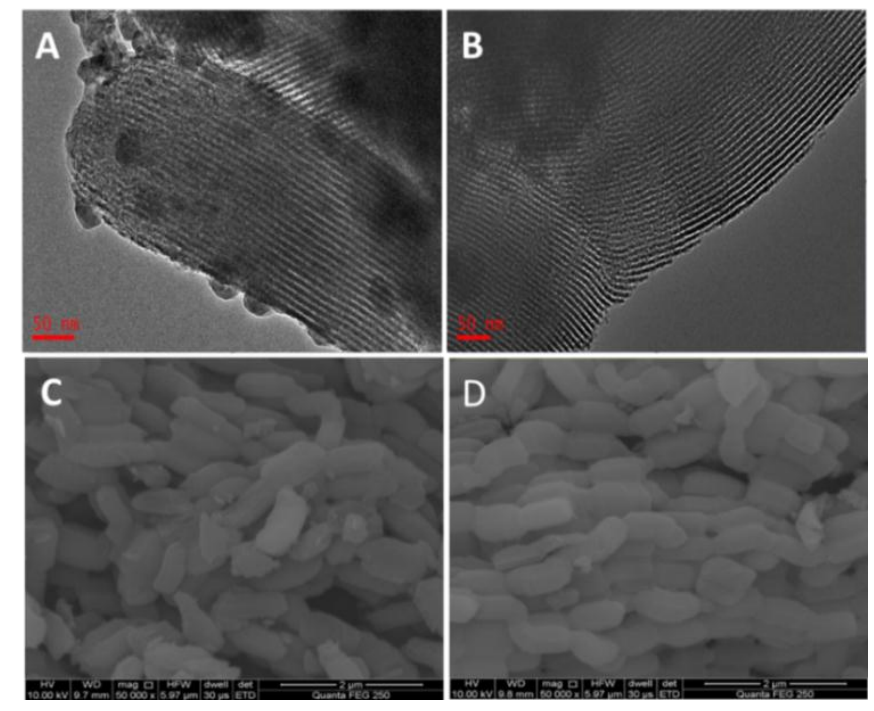

Fig. 2. (A) HRTEM image and (C) SEM image of Ce-SBA-15, (B) HRTEM image and (D) SEM image of Al-Ce-SBA-15. 
The $\mathrm{N}_{2}$ absorption-desorption isotherms, pore size distributions and the textural properties of SBA-15, Ce-SBA-15 and Al-Ce-SBA-15 as shown in Fig. 3 and Table 1. The isotherms of all the samples give a clear $\mathrm{H} 1$ type hysteresis loop at high relative pressure, which suggests that the samples have very regular mesoporous channels of SAB-15 as shown in Fig. 3A. This is also evident by the BJH pore diameter distributions in Fig. 3B which showed a narrow pore size distribution. It can be seen that the BET surface area, pore volume and pore diameter of Ce-SBA-15, Al-Ce-SBA-15 slightly decreased as compared with SBA-15 in Table 1, which might be associated with the formation of non-framework $\mathrm{Ce}$ and $\mathrm{Al}$ species in the mesoporous channels. These $\mathrm{N}_{2}$ adsorption-desorption data are in very good agreement with the results of XRD, TEM and SEM.
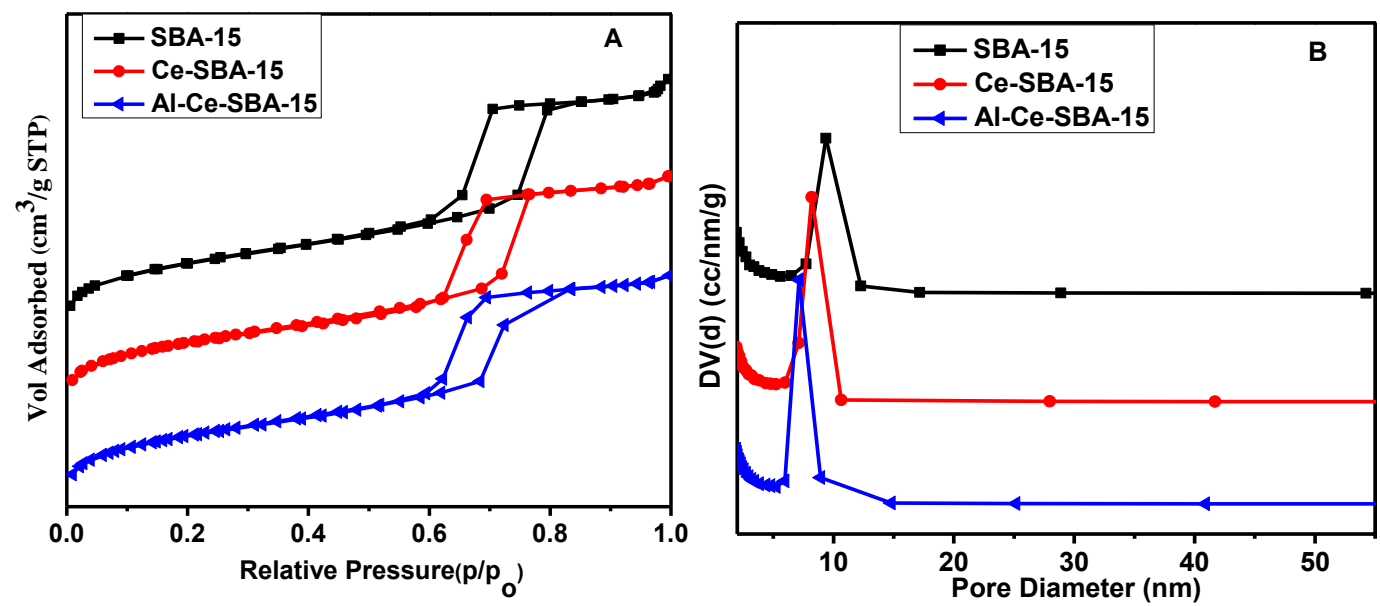

Fig 3. Nitrogen adsorption-desorption isotherms (A) and pore diameter distribution (B) of SBA-15, Ce-SBA-15, Al-Ce-SBA-15.

Table 1

Textural properties of the samples

\begin{tabular}{lccccc}
\hline Sample & $\begin{array}{c}\mathrm{d}_{100} \\
(\mathrm{~nm})\end{array}$ & $\begin{array}{c}\text { Surface area } \\
\left(\mathrm{m}^{2} / \mathrm{g}\right)\end{array}$ & $\begin{array}{c}\text { Pore diameter } \\
(\mathrm{nm})\end{array}$ & $\begin{array}{c}\text { Pore volume } \\
\left(\mathrm{cm}^{3} / \mathrm{g}\right)\end{array}$ & $\begin{array}{c}\mathrm{a}_{0} \\
(\mathrm{~nm})\end{array}$ \\
\hline SBA-15 & 9.2 & 810 & 6.6 & 0.95 & 10.6 \\
Ce-SBA-15 & 9.3 & 789 & 6.0 & 0.89 & 10.7 \\
Al-Ce-SBA-15 & 9.0 & 773 & 5.8 & 0.87 & 10.4 \\
\hline
\end{tabular}

Pore diameter and pore volume calculated from the $\mathrm{N}_{2}$ desorption data based on the $\mathrm{BJH}$ method 
$\mathrm{a}_{0}=2 \mathrm{~d}_{100} / \sqrt{3}$.

The performance of Al-Ce-SBA-15 material was further investigated by introduced a strong base guest of KF with impregnation method, and then the solid basic KF/Al-Ce-SBA-15 catalyst was prepared by calcination. Meanwhile, the catalytic performance of KF/Al-Ce-SBA-15 catalyst was tested for the synthesis of propylene glycol methyl ether (PGME) from methanol with propylene oxide (PO). For comparison, the KF/SBA-15 and KF/Ce-SBA-15 were synthesized as the same method. The catalytic performance of the samples SBA-15, KF/SBA-15, Al-Ce-SBA-15, KF/Ce-SBA-15 and KF/Al-Ce-SBA-15 were illustrated in Table.S1, which showed that the KF/Al-Ce-SBA-15 has higher catalytic activity compared to the other samples.

\section{Conclusion}

A kind of ordered mesoporous material Al-Ce-SBA-15 wase successfully prepared by the method of dual-templates CTAB and P123 with adding appropriate amount of Al through a one-step hydrothermal synthesis. The results of XRD, EDX, BET, HRTEM, SEM and FT-IR showed that the heteroatoms Al and Ce highly dispersed on the surface of SBA-15 and the prepared material of Al-Ce-SBA-15 has a highly ordered mesoporous structure, high surface areas $\left(773 \mathrm{~m}^{2} / \mathrm{g}\right)$, large pore volumes $\left(0.87 \mathrm{~cm}^{3} / \mathrm{g}\right)$ and narrow pore size distribution. More significantly, a uniform coating layer was formed on the surface of SBA-15, and the aggregation of metal Ce was avoided by adding appropriate amount of Al.

\section{Acknowledgements}

The authors gratefully the financial support from the National Science Foundation of China (No. 21276217), the Graduate Innovation Fund of Hunan province (No. CX2013B276), the China Postdoctoral Science Foundation funded project (No. 2014M552143) and Hunan Provincial Department of Education Fund (No. 13C912).

\section{References}

[1] Kresge CT, Leonowicz ME, Roth WJ, et al. nature 1992; 359:710-712.

[2] Ding Y, Yin G, Liao X, et al. Micropor Mesopor Mater 2013; 170: 45-51. 
[3] Alfredsson V, Wennerström H. Accounts Chem Res 2015; 48: 1891-1900.

[4] Hunt AJ, Budarin VL, Comerford JW, et al. Mater Lett 2014; 116: 408-411.

[5] Yi Q, Zhang J, Zhang X, et al. Fuel 2015; 143: 390-398.

[6] Sun LB, Sun YH, Liu XD, et al. Curr Org Chem 2014; 18: 1296-1304.

[7] Zhu L, Lu F, Liu XD, et al. Chem Commun 2015; 51:10058-10061.

[8] Sun YH, Sun LB, Li TT, et al. J Phys Chem C 2010; 114: 18988-18995.

[9] Wu ZY, Jiang Q, Wang YM, et al. Chem mater 2006; 18: 4600-4608.

[10] Sauer J, Marlow F, Spliethoff B, et al. Chem Mater 2002; 14: 217-224.

[11] Wang N, Chu W, Zhang T, et al. Int J Hydrogen Ener 2012; 37: 19-30.

[12] Ono H, Katsumata T. Appl Phys Lett 2001; 78: 1832-1834.

[13] Sauer J, Marlow F, Schüth F. Phys Chem Chemi Phy 2001; 3: 5579-5584.

[14] Dai Q, Wang X, Chen G, et al. Micropor Mesopor Mater 2007, 100: 268-275.

[15] Thitsartarn W, Maneerung T, Kawi S. Energy 2015; 89: 946-956.

[16] Zhao D, Feng J, Huo Q, et al. Science 1998; 279: 548-552. 
Graphical abstract: The ordered mesoporous material Al-Ce-SBA-15 was synthesized by the method of dual templates CTAB and P123 with adding appropriate amount of Al.

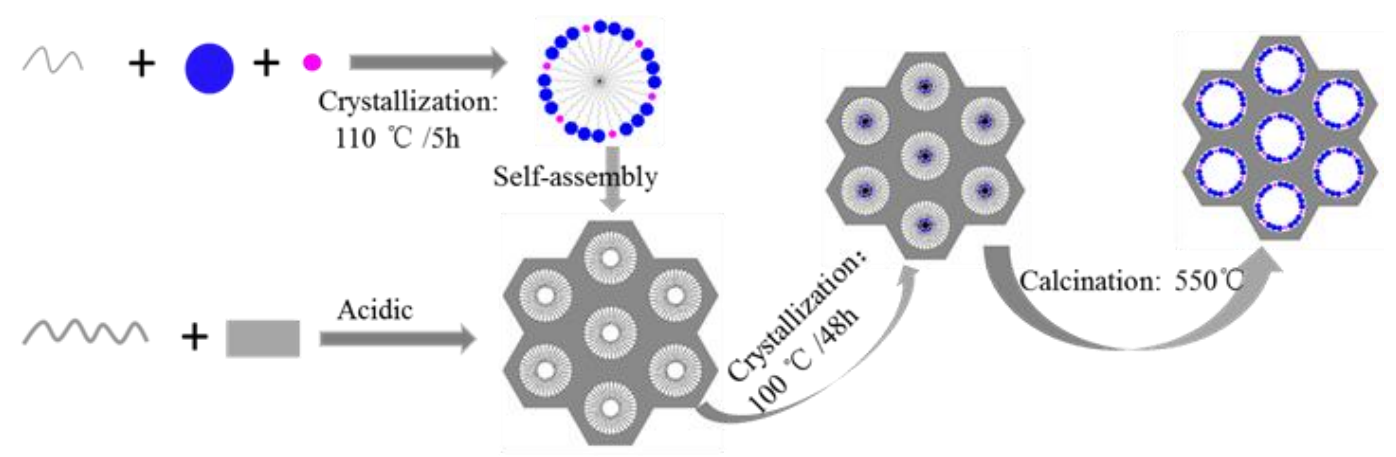

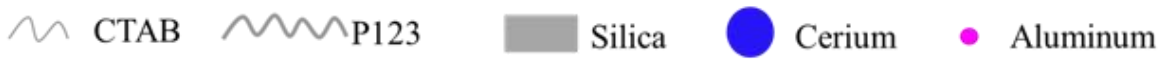

